\title{
Extracellular potassium concentration defines neuronal bursting properties
}

\author{
Yaroslav I Molkov ${ }^{1 *}$, Bartholomew J Bacak², Joshua Segaran³, llya A Rybak² \\ From 24th Annual Computational Neuroscience Meeting: CNS*2015 \\ Prague, Czech Republic. 18-23 July 2015
}

Many neurons, or populations of neurons, in the brain are capable of producing rhythmic bursting activity. This ability is putatively responsible for rhythmogenic functions like breathing and locomotion. In vivo, rhythms are generated by synaptically interconnected neuronal networks, whereas rhythmic bursting behavior is often induced in vitro by elevating the extracellular potassium concentration $\left(K_{\text {out }}\right)$ [1]. It is known that increasing $K_{\text {out }}$ raises the reversal potentials of potassium and leak currents [2]. However, the complete nature of how these effects underlie bursting activity has yet to be uncovered.

A mathematical modeling study was performed to elucidate the interplay between these factors and their roles in a neuron's transition from quiescence to rhythmic bursting. A conductance-based model of a neuron from the pre-Bötzinger Complex (pre-BötC) was used as a basis [3]. A potassium ion component was incorporated into the leak current, and model behaviors were investigated at varying concentrations of $K_{\text {out }}$, taking into account its effect on delayed rectifier potassium current responsible for after-spike hyperpolarization. The primary aim of this modeling study was to evaluate the contribution of extracellular potassium ions in the leak and delayed rectifier potassium current, and the subsequent effect of these altered currents on the bursting properties of neurons. Furthermore, the initial model was modified to replicate experimental results and test for conditions of low $K_{\text {out }}$ as seen in vivo.

The analysis of our model shows that: (i) in vitro bursting behavior with elevated $K_{\text {out }}$ may occur due to attenuation of the delayed rectifier potassium current and (ii) no oscillations are generated at physiological

\footnotetext{
* Correspondence: ymolkov@iupui.edu

'Department of Mathematical Sciences, Indiana University-Purdue University, IN, USA

Full list of author information is available at the end of the article
}

levels of extracellular potassium. These results indicate that, according to the commonly-accepted models used in our study, neurons that naturally burst in in vitro preparations may not be able to burst in vivo under any circumstances. Accordingly, rhythmic activity in vivo should rely on other mechanisms. For example, Jasinski et al. [4] have shown that the recurrent synaptic excitation in combination with the sodium-potassium exchanger (pump) can result in the robust rhythmic network activity even with all intrinsic bursting mechanisms blocked.

\section{Acknowledgements}

This work was supported by National Institutes of Health, grant R01 AT008632 to Y. I. M.; grants R33 HL087377; R01 NS057815; and R01 NS069220 to I. A. R.

\section{Authors' details}

Department of Mathematical Sciences, Indiana University-Purdue University, IN, USA. ${ }^{2}$ Department of Neurobiology and Anatomy, Drexel University College of Medicine, PA 19123, USA. ${ }^{3}$ Carmel High School, Carmel, IN 46032, USA.

Published: 18 December 2015

\section{References}

1. Smith JC, Ellenberger HH, Ballanyi K, Richter DW, Feldman JL: Pre-Botzinger Complex - a Brain-Stem Region That May Generate Respiratory Rhythm in Mammals. Science 1991, 254(5032):726-729.

2. Rybak IA, Shevtsova NA, St-John WM, Paton JFR, Pierrefiche O: Endogenous rhythm generation in the pre-Botzinger complex and ionic currents: modelling and in vitro studies. Eur J Neurosci 2003, 18(2):239-257.

3. Butera RJ, Rinzel J, Smith JC: Models of respiratory rhythm generation in the pre-Botzinger complex. I. Bursting pacemaker neurons. I Neurophysiol 1999, 82(1):382-397.

4. Jasinski PE, Molkov YI, Shevtsova NA, Smith JC, Rybak IA: Sodium and calcium mechanisms of rhythmic bursting in excitatory neural networks of the pre-Botzinger complex: a computational modelling study. Eur J Neurosci 2013, 37(2):212-230.

doi:10.1186/1471-2202-16-S1-P215

Cite this article as: Molkov et al:: Extracellular potassium concentration defines neuronal bursting properties. BMC Neuroscience 2015 16(Suppl 1): P215. 